\title{
A wireless networking architecture using MANET for mobile communications of remote pastoral areas in Tibet
}

\author{
Cao Huang \\ School of Information Engineering \\ Tibet University for Nationalities \\ Xianyang, Shaanxi, China \\ caohuang@ustc.edu
}

\author{
Xiaojun Guo \\ School of Information Engineering \\ Tibet University for Nationalities \\ Xianyang, Shaanxi, China \\ gxj_0617@163.com
}

\author{
Zeguo Liu \\ School of Information Engineering \\ Tibet University for Nationalities \\ Xianyang, Shaanxi, China \\ zeguo_liu@163.com
}

\begin{abstract}
To support mobile communications in remote pastoral areas of Tibet effectively, this paper proposes an integrated wireless networking architecture using MANET. The sparse mobile users, assisted by the solar-powered multifunctional standing station mainly on networking maintenance and routing arrangement, self-organize into a MANET. The elements of the architecture are described specifically. The proposed architecture can help to reduce the network deployment cost and provide the mobile users voice, message and low rate data services.
\end{abstract}

Keywords-MANET; mobile communication; networking; architecture; Tibet

\section{INTRODUCTION}

In remote rural and pastoral areas of Tibet, some certain mobile communications needs have not been meted due to the lack of certain economic convenient mobile communication tools. Mainly, the nomadic herdsmen demand mobile communications during the grazing season. Secondly, there are some mobile communication demands for scientific investigating tasks and emergency missions. With the economic and social development of Tibet and the country, these demands need to be satisfied more and more urgently [1]. But to meet these needs through the expansion of existing cellular systems (including 2G and $3 G$ ) is extremely uneconomical, because these requirements are not only relatively sparsely distributed in a very vast land but also seasonal or unexpected. Another possible solution is to use satellite communications, but the several satellite systems for personal communication use charge too much to afford. To address the faced problem, we propose to build a networking architecture using the MANET (Mobile Ad hoc Network) concept in this paper.

A MANET is a 'temporary' self-configuring multi-hop network of mobile devices connected by wireless, which does not rely on available fixed infrastructure. Each device in a MANET is free to move independently in any direction, and will therefore change its links to other devices frequently. Each is equipped to continuously maintain the information required to properly route traffic. Such networks may operate by themselves or may be connected to the larger Internet. So to network using MANET is flexible, convenient and rapid.
At present, it is mainly used in military tactics communication and emergency communication field. In recent years, along with the mobile equipment miniaturization and construction cost lowering, MANET has begun to participate in personal communication network establishment and to be becoming an important form of network accessing [2][3].

To establish a MANET networking architecture for mobile communications of remote pastoral areas in Tibet, there are two main technical problems need to be resolved:

- The mobile users are so scarcely distributed that they can not self-network and connect directly with other ground communication system.

- The mobile users' devices are extremely energyconstraint, though the nomadic herdsmen would carry a small solar power plant (or mixed with a small wind power unit) with one or two solar panels.

Several frequency bands could be selected for Ad hoc systems. Considering the sparse distribution of the mobile users and the very energy-constraint, the MANET system will just satisfy voice, message, and low-rate data needs, and the proper frequency range will be around $300 \mathrm{MHz}$ or 450MHz-500MHz.

The rest of the paper is organized as follows. Section II proposes how MANET is integrated with the cellular network system. In Section III, how the network elements are organized and their functionalities are described specifically. Section IV gives a description on the crosslayer structure for the MANET system. Section V concludes this paper.

\section{MANET CONNECTED WITH CELLULAR NETWORK}

In the remote pastoral areas in Tibet, there exit areas of coverage of the cellular system base station only near the very main roads. When the mobile users are within the radio coverage of cellular network, they just use the available system. But while they are standing far away, they will selforganize into a MANET as showed in Fig.1. The MANET between mobile users is the transmission network for userto-user communications, and also acts as the transit network for user-to-cellular-network communications.

Supported by the Key Project of Chinese Ministry of Education.(No.212168) 


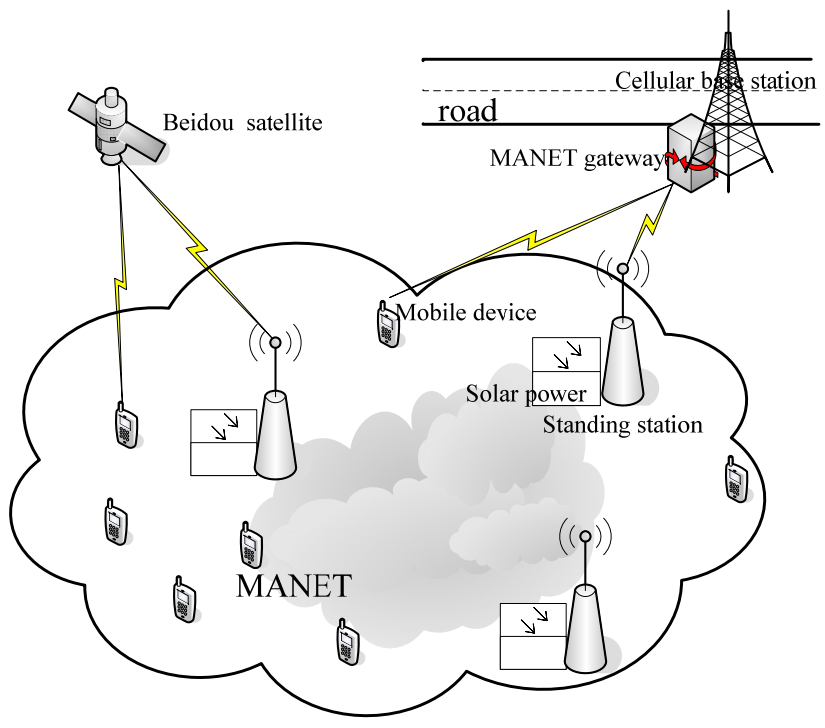

Figure 1. MANET networking architecture

As showed in Fig.2, the MANET connects with the cellular network via some terrestrial gateway working at the network layer, which could be built within some cellular base stations. The coverage of the gateway's antenna is much more than that of the cellular base station. The gateways receive the wireless signals from the MANET, and transmit the contained information to the cellular base station. Then, The MANET connects with Internet via the cellular network gateway.

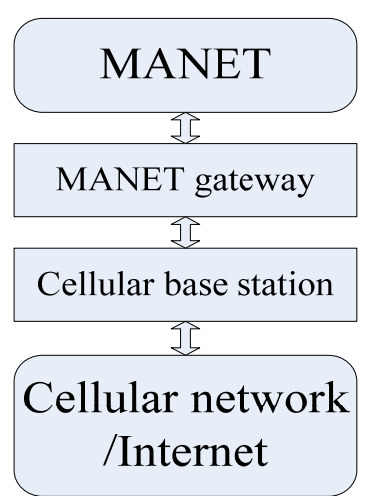

Figure 2. MANET connected with cellular network

The MANET gateway is an authorizing center and an IP proxy. Though an all-IP network and IP mobility management is a general trend [4], it is not very necessary in this MANET due to the increased overheads and complexity. The MANET gateway (plus the standing stations) only admits the authorized devices to access.

\section{MANET ELEMENTS AND FUNCTIONS}

In the MANET as showed in Fig.1, there are two types of nodes. One is the standing station node; the other is the mobile user device node. The two types of nodes both participate in self-organizing network, but does not work as same as the common clustering Ad hoc network. The followings describe their characteristics and functions specifically.

\section{A. The Standing Station}

In the MANET, the standing stations have two functions. When the few mobile devices are so scarcely distributed that they can not network on their own, the standing stations help them to connect with each other and with the cellular gateway. In the other situation, when the mobile devices can network by themselves, both the two kinds of nodes are together self-organizing into a MANET but play different roles. The stations will play a more important role on networking maintenance and routing arrangement. The management and the routing methods are expected to be based on energy control, position and hierarchy information. In general, the stations will adapt data routing paths according to their energy status. To preserve nodes surviving is a priority.

The standing station is supported by solar energy, since Tibet is one of those distracts that have best solar energy. When the solar power is most powerful in summer, the station will be most utilized because it is the season for nomadic herdsmen and best season for all other kinds of activities. So there is no need to install wire infrastructure, which will cost too much. The stations have CNSS (Compass Navigation Satellite System, also called Beidou [5]) terminals for positioning and clocking. They are unwatched, and will be examined and maintained each summer as required.

Different from the omni-directional antenna used by the mobile users' devices, three or four directional antennas will be equipped at the station. Due to these antennas and the solar energy, one station's signals can cover a much vast area.

The standing stations are supposed to inter-connect with each other. Fig. 3 shows the ideal topology of the standing stations. Each station connects with other three stations directly except the ones nearest to the MANET gateways. The coverage of the MANET gateway is much more than that of the cellular base station, and each gateway could connect with two or three the standing stations directly. The real topology will be some deformed of the ideal one according to the real topography, but the relation of connectivity just mentioned is unchanged, which makes sure that when one station losses connection with two stations, it will still in the station network. Furthermore, when several stations are not in operation, the station network will still be a connected graph. 


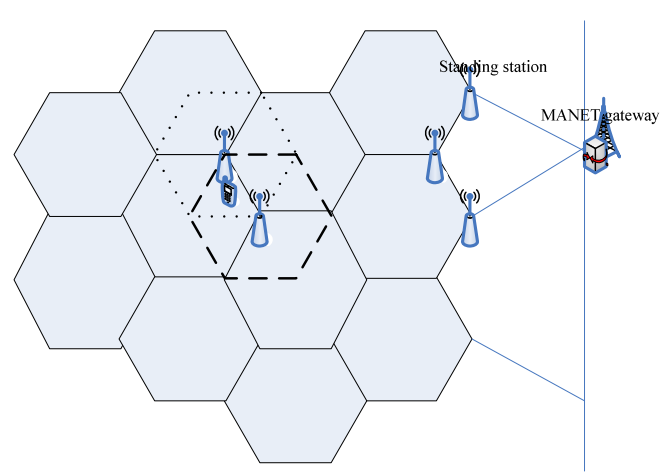

Figure 3. Ideal topology of standing stations

Each mobile user device is in the coverage of two standing stations as shown in Fig.3 using two dotted lines. The mobile user could select one to connect according to the status of energy and busyness of the standing stations. If one station is broken, there still exits one to connect.

It is worth to note that the standing station is multifunctional, and the standing station network is supposed to be clustering node network for wireless sense networks. The station network with the wireless sense networks can do environmental sensing and deliver the sensing information back all the year, which is very important for Tibet environment research and protect. Also, the stations may be equipped with many other functions, such as being a power supplier of small electronic devices for passers-by, and so on.

\section{B. The Mobile User Device}

The mobile device is multi-accessing-modes integrated. Three accessing modes are provided, respectively with cellular network, MANET and Beidou satellites.

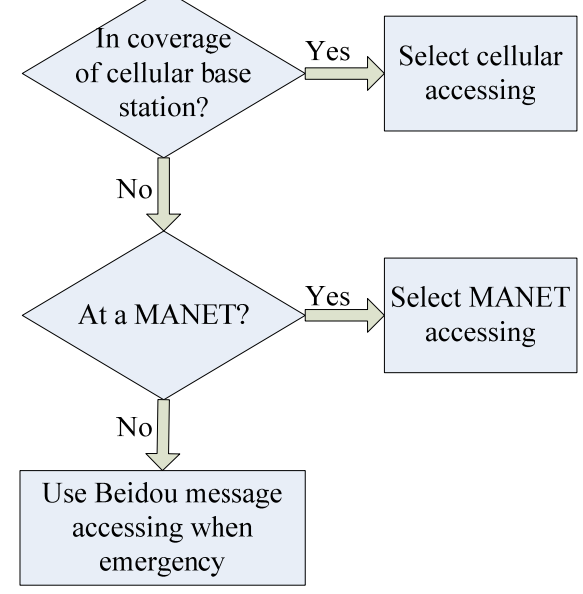

Figure 4. Procedure of accessing selection for mobile user device

Fig. 4 shows the procedure on how the mobile user device does select an accessing.

Firstly, when it is under the coverage of the cellular base station, it can connect directly to the base station using the 2G/3G cellular access. To be noted that when a mobile device is in the coverage of some cellular base station, it may also be in one MANET's. In this situation, the mobile device turns off the MANET accessing.

The secondary is the MANET access, which provides communications between MANET mobile users or with Internet through the gateways.

Lastly, the mobile user devices are also equipped with Beidou terminals. So, when some mobile users have emergency information and the other accessings can not be used, they can send messages through the Beidou message service.

\section{MANET CROSSLAYER STRUCTURE}

It is worth noting that the mobile user device is energyconstraint. Though the nomadic herdsmen would carry a small solar power plant (or mixed with a small wind power unit) with one or two solar panels, the energy for the device is always a scarce resource.

How to reduce packet droppings, data retransmissions and control overheads is the key not only for conserving energy but also for improving MANET system efficiency.

The traditional five layer protocol model can not adapt to the wireless mobile environment well. For example, the current TCP implicitly assume that packet droppings are all come from congestions. But in this situation, packet droppings may caused by unstable wireless channels, which will make the TCP data rate much low and inefficiency.

So the crosslayer method is designed for the MANET system as shown in Fig.5.

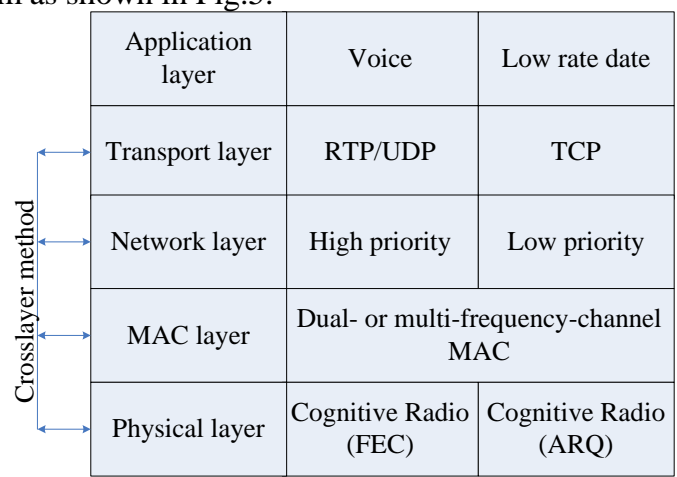

Figure 5. Crosslayer structure for different services

First of all, to satisfy different Qos of different services, different network layer and physical layer solutions are provided. For voice service, the high priority at network layer makes voice latency lower and acceptable. And FEC (forward error correction) coding is used to reduce noise. Comparatively, for low rate data service, Selected-AQR (Automatic Repeat Request) decreases the bad influence of channel droppings to the TCP.

Cognitive radio is a developing technology originating form software defined radio, which could detect wireless channel environment dynamically and utilize available frequency bands efficiently. In [6], a cognitive radio-based multi-channel MAC protocol is proposed for Ad hoc networks, which is a good reference to draw for this MANET system. 
More complex issue is the routing protocol. The expected crosslayer routing protocol would interact with the cognitive radio layer and should base on energy control, position and hierarchy information [7][8][9].

\section{CONLUSION}

In this paper, we propose an integrated wireless networking architecture using MANET for mobile communications of remote pastoral areas in Tibet, which can reduce the network deployment cost. The sparse mobile users, assisted by the solar-powered multi-functional standing station mainly on networking maintenance and routing arrangement, self-organize into a MANET. The MANET connects with the cellular network via the MANET gateway, and with Internet via the cellular network.

The MANET provides voice, message and low-rate data services to the mobile user. Besides the MANET accessing, the mobile user devices have cellular accessing and Beidou message accessing to select for different situations, which makes sure that the mobile users get maximum communication guarantee.

A framework of crosslayer structure is proposed to enhance the system efficiency and conserve energy. The specific crosslayer MAC protocol and routing protocol need to be constructed and are the next research focus for the proposed MANET architecture.

\section{REFERENCES}

[1] Report of the People's Government of the Tibet Autonomous Region on accelerating the development of the communications industry. Tibet Government Report No. 37 [2011]

[2] I F. Akyildiz, Xd Wang et al, "Wireless mesh networks: a survey". Computer Networks, vol.47, 2005, pp. 475-487

[3] WC Du, Zhx Ma, Y Bai et al, "Integrated Wireless Networking Architecture for Maritime Communications”, 11th ACIS, 2010, pp. 134-138

[4] F M. Abduljalilh and S K. Bodhe, "A survey of integrating IP mobility protocols and mobile Ad hoc networks", IEEE Communications Surveys \& Tutorials, 2007, Vol.9, No.1, pp. 14-30

[5] http://www.beidou.gov.cn/

[6] J Min, Lj Ge, “a cognitive radio-based multi-channel MAC protocol for ad hoc networks", Computer Applications and Software, 2010, Vol.27, No.11, pp.223-225

[7] H Cheng, J Cao, “A Design Framework and Taxonomy for Hybrid Routing Protocols in Mobile Ad Hoc Networks", IEEE Communications Surveys \& Tutorials, 2008, Vol.10, No.3, pp.62-73

[8] K Wen, W Guo, and GJ Huang, "Topology-Based Power-Aware Routing Protocol in the Wireless Ad hoc Networks", Journal of Electronics \& Information Technology, 2008, Vol.30, No.12, pp. 3013-3017

[9] Lb Zhai, Y-a Liu, “A Tree-based Cognitive Radio Routing Protocol in Ad Hoc Networks", Journal of Beijing University of Posts and Telecommunications, 2012, Vol.35, No.1, pp.85-89 